PROCEEDINGS OF THE

AMERICAN MATHEMATICAL SOCIETY

Volume 130, Number 1, Pages 243-252

S 0002-9939(01)06000-

Article electronically published on May 7, 2001

\title{
ON UNKNOTTING NUMBERS AND FOUR-DIMENSIONAL CLASP NUMBERS OF LINKS
}

\author{
TOMOMI KAWAMURA
}

(Communicated by Ronald A. Fintushel)

\begin{abstract}
In this paper, we estimate the unknotting number and the fourdimensional clasp number of a link, considering the greatest euler characteristic for an oriented two-manifold in the four-ball bounded by the link. Combining with a result due to Rudolph, we prove that an inequality stronger than the Bennequin unknotting inequality actually holds for any link diagram. As an application we show the equality conjectured by Boileau and Weber for a closed positive braid diagram.
\end{abstract}

\section{InTRODUCTION AND THE STATEMENTS OF MAIN RESUltS}

A link is a closed oriented 1-manifold smoothly embedded in the 3 -sphere $S^{3}$ and a knot is a link with one component. Let $L$ be a link with $r$ components. The unknotting number $u(L)$ of the link $L$ is the minimal number of crossing changes needed to create the trivial link with $r$ components.

It is well known that the unknotting number is not less than the 4-genus [19]. The 4-genus $g^{*}(L)$ of the link $L$ is the minimum genus for an oriented 2-manifold, without closed component, which is smoothly embedded in 4-ball with boundary $L$. In this paper we consider the following link invariants to give lower bounds for the unknotting numbers. Let $L$ be a link and $F \subset D^{4}$ a smooth, oriented 2-manifold with $\partial F=L$, where $D^{4}$ is the 4 -ball bounded by $S^{3}$. We suppose that $F$ has no closed components, but $F$ is not assumed to be connected. We denote by $\chi_{s}(L)$ the greatest value of the euler characteristic $\chi(F)$ for such 2-manifolds $F \subset D^{4}$. We denote by $c_{s}(L)$ the minimum number of the double points for transversely immersed disks in $D^{4}$ with boundary $L$ and with only finite double points as singularities, and we shall call this invariant the 4-dimensional clasp number. We have the following relations among the unknotting number, the clasp number, and the euler characteristic $\chi_{s}(L)$.

Theorem 1.1. Let $L$ be an oriented link with $r$ components. Then the following inequality holds:

$$
u(L) \geq c_{s}(L) \geq \frac{1}{2}\left(r-\chi_{s}(L)\right) .
$$

Received by the editors October 4, 1999 and, in revised form, May 12, 2000.

2000 Mathematics Subject Classification. Primary 57M25.

Key words and phrases. Unknotting number, 4-dimensional clasp number, Bennequin unknotting inequality.

The author was partially supported by JSPS Research Fellowships for Young Scientists. 


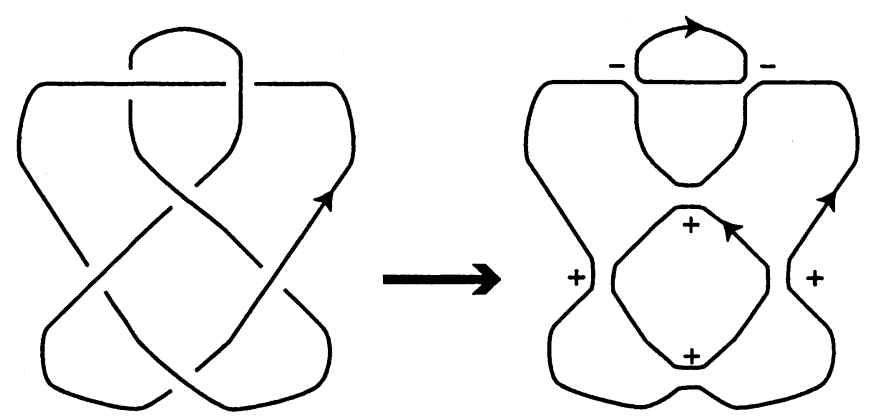

Figure 1. A link diagram and Seifert circles

Let $D$ be an oriented link diagram. The writhe $w(D)$ is the value defined by the number of positive crossings minus the number of negative crossings of $D$. We give signs to Seifert circles of $D$ as follows. A Seifert circle is a strongly negative circle if it is adjacent to at least two negative crossings but adjacent to no positive crossings. A Seifert circle is a non-negative circle if it is not a strongly negative circle.

For example, the trivial diagram of a trivial knot has a non-negative circle. The blackboard diagram of a (-1)-framed trivial knot has two non-negative circles. A knot diagram illustrated on the left of Figure 1has three Seifert circles as illustrated on the right. The top circle is strongly negative and the concentric circles at the bottom are non-negative.

By means of Theorem 1.1 and a result announced by Rudolph [18], that is, Theorem 3.1, we obtain the following inequality.

Corollary 1.2. Let $L$ be an oriented link with $r$ components and $D_{L}$ any diagram for $L$. Let $s_{\geq}\left(D_{L}\right)$ be the number of non-negative circles of $D_{L}$ and $s_{<}\left(D_{L}\right)$ the number of strongly negative circles. Then we have

$$
u(L) \geq c_{s}(L) \geq \frac{1}{2}\left\{r-\left(s_{\geq}\left(D_{L}\right)-s_{<}\left(D_{L}\right)\right)+w\left(D_{L}\right)\right\} .
$$

Let $s(D)$ be the number of the Seifert circles of a link diagram $D$. Let $L$ be an $r$-components link which has a closed braid diagram $D_{L}$. In [2], Bennequin conjectured the inequality

$$
u(L) \geq \frac{1}{2}\left(r-s\left(D_{L}\right)+\left|w\left(D_{L}\right)\right|\right) .
$$

We shall call it the Bennequin unknotting inequality. In [16] Rudolph showed the slice-Bennequin inequality

$$
\chi_{s}(L) \leq s\left(D_{L}\right)-w\left(D_{L}\right) .
$$

It implies the Bennequin unknotting inequality by means of Theorem 1.1 However, to the best of my knowledge, the complete proof of the Bennequin unknotting inequality had not been written. In [20], Stoimenow showed that if the Bennequin unknotting inequality holds for any closed braid diagram, then it holds for any knot diagram. In fact, both of the Bennequin unknotting inequality and the sliceBennequin inequality can be extended to any link diagram by the Vogel algorithm [22] or the Yamada algorithm [23]. Corollary 1.2 shows that an inequality stronger than that conjectured by Bennequin actually holds. 
A closed positive braid diagram is a closed braid diagram with no negative crossings. Let $L$ be an $r$-components link which has a closed positive braid diagram $D_{L}$. Let $x\left(D_{L}\right)$ be the number of the crossings of $D_{L}$. Boileau and Weber 3 conjectured the equality

$$
u(L)=\frac{1}{2}\left(r-s\left(D_{L}\right)+x\left(D_{L}\right)\right) .
$$

By means of Corollary 1.2, we determine not only the unknotting numbers but also the 4-dimensional clasp numbers of closed positive braids.

Theorem 1.3. Let $L$ be an oriented link with $r$ components. We suppose that $L$ has a closed positive braid diagram $D_{L}$. Let $s\left(D_{L}\right)$ be the number of the Seifert circles and $x\left(D_{L}\right)$ the number of the crossings of $D_{L}$. Then we have

$$
u(L)=c_{s}(L)=\frac{1}{2}\left(r-s\left(D_{L}\right)+x\left(D_{L}\right)\right) .
$$

In the second section, we explain the 4-dimensional clasp number and prove Theorem 1.1. In the third section, we prove Corollary 1.2 and Theorem 1.3. In order to prove Corollary 1.2 we reprove an inequality announced by Rudolph [18]. In the last section of this paper, we give two examples as applications of the arguments of the above results.

\section{FOUR-DIMENSIONAL CLASP NUMBERS}

In this section, we prove the well-definedness of the 4-dimensional clasp number and prove Theorem 1.1

Lemma 2.1. For any link $L$, there are transversely immersed disks in $D^{4}$ with boundary $L$ and with only finite double points as singularities.

Proof. We suppose that $L$ is unknotted by $u$-crossing changes and that $L$ has $r$ components. We construct suitable immersed disks in $D^{4}$ with boundary $L$ by this unknotting operation. We suppose that $L$ is a link in $S^{3} \times\{0\}$ and let $O_{r}$ be a trivial link with $r$ components in $S^{3} \times\{1\}$. The trivial link $O_{r}$ bounds $r$ disks $D_{r}$ in $S^{3} \times\{1\}$. There exist proper immersed annuli $F_{0}$ in $S^{3} \times[0,1]$ such that $\partial F_{0}=L \cup O_{r}$ and $F_{0}$ has $u$ double points. We can consider that

$$
F_{0} \cup_{O_{r}} D_{r} \subset S^{3} \times[0,1]=\partial D^{4} \times[0,1] \subset D^{4}
$$

is immersed disks in $D^{4}$ with $u$ double point.

In [19, Shibuya defined the invariant $c^{*}(L)$ as the minimum number of the double points for a transversely immersed surface with genus 0 and boundary $L$. He called $c^{*}$ the clasp number. From definitions, $c^{*}(L) \leq c_{s}(L)$. But the equality does not hold in general, because if $L$ is the Hopf link, $c^{*}(L)=0$ and $c_{s}(L)=1$. But the argument for the well-definedness of $c^{*}$ in [19] is similar to the one of $c_{s}$. We note that $c_{s}$, the 4 -dimensional clasp number defined in previous section, does not depend on the orientations of links, and is invariant under an orientation-reversing diffeomorphism of $S^{3}$.

Proof of Theorem 1.1. By the argument in the proof of Lemma 2.1, we have $u(L) \geq$ $c_{s}(L)$. We prove $c_{s}(L) \geq\left(r-\chi_{s}(L)\right) / 2$.

Let $F^{\prime}$ be immersed disks in $D^{4}$ with boundary $L$ and exactly $c$ double points as singularities. A small neighborhood of each of such double points in $F^{\prime}$ is a cone over the Hopf link in the small 4-ball. In the boundary of the small 4-ball, this 
Hopf link bounds an annulus. We replace the small neighborhoods of those double points in $F^{\prime}$ with such $c$ annuli. Then we obtain new 2 -submanifold $F \subset D^{4}$. We may suppose that $F$ is a smooth, oriented 2-manifold such that $L=\partial F$. $F$ has no closed components. Then we have

$$
\begin{aligned}
\chi_{s}(L) & \geq \chi(F) \\
& =\chi((r \text { disks })-(2 c \text { small disks }))+c \chi(\text { annulus })-2 c \chi(\text { circle }) \\
& =r-2 c .
\end{aligned}
$$

Hence the following inequality holds:

$$
c_{s}(L) \geq \frac{1}{2}\left(r-\chi_{s}(L)\right) .
$$

In [18, Rudolph commented on the inequality $u(L) \geq\left(r-\chi_{s}(L)\right) / 2$ for $r=1$. If $r=1$, that is, $L$ is an oriented knot, the equality $g^{*}(L)=\left(r-\chi_{s}(L)\right) / 2$ holds. We note that it may not hold if $r \geq 2$. In fact, in the case of the Hopf link, the 4-genus is 0 and $\chi_{s}(L)$ is 0 .

In 19], Shibuya proved $g^{*} \leq c^{*} \leq u$ for any link. The argument in the proof of Theorem 1.1 is similar to his argument. If $r=1, c^{*}(L)=c_{s}(L)$ holds. In [13], Murakami and Yasuhara proved that the 4-dimensional clasp number of the knot $8_{16}$ is 2 and not equal to $g^{*}\left(8_{16}\right)=1$. So, the equality $c_{s}(L)=\left(r-\chi_{s}(L)\right) / 2$ does not hold in general. The existence of non-trivial slice knots implies that the equality $u(L)=c_{s}(L)$ does not hold in general.

Remark 2.2. Let us compare the 4-dimensional clasp number with the kinkiness of the knot defined by Gompf 4 . Let $k_{+}(K)$ and $k_{-}(K)$ be the minimum number of positive double points and the minimum number of negative double points for a transversely immersed disk in the 4 -disk bounded by the knot $K$ respectively. The kinkiness of the knot $K$ is the pair $k(K)=\left(k_{+}(K), k_{-}(K)\right)$. From definitions, we have $c_{s}(K) \geq k_{+}(K)+k_{-}(K)$.

\section{The Bennequin unknotting inequality}

To prove Corollary 1.2 , we use the following theorem announced by Rudolph:

Theorem 3.1 (Rudolph[18]). Let $L$ be an oriented link and $D_{L}$ any diagram for $L$. Let $s_{\geq}\left(D_{L}\right)$ be the number of non-negative circles of $D_{L}$ and $s_{<}\left(D_{L}\right)$ be the number of strongly negative circles. Then

$$
\chi_{s}(L) \leq\left(s_{\geq}\left(D_{L}\right)-s_{<}\left(D_{L}\right)\right)-w\left(D_{L}\right) .
$$

Since he proved only for the case where the $D_{L}$ has the at least one positive crossing in [18, we reprove this theorem.

In the proof of Theorem 3.1, we use results by Nakamura [14] and Rudolph [16, [18]. In [14], [18, they proved that positive links are quasipositive. A quasipositive link is the link which has a closed quasipositive braid diagram, and a quasipositive braid is the product of conjugate braids with positive braids. In [15, Rudolph showed that such a link appears as the intersection of an embedded 3-sphere and an algebraic curve in the 2-dimensional complex plane. Let $s(D)$ be the number of the Seifert circles of a link diagram $D$. In [16], Rudolph showed that the equality $\chi_{s}(L)=s\left(D_{L}\right)-w\left(D_{L}\right)$ holds for any closed quasipositive braid diagram $D_{L}$ by the so-called generalized adjunction formula. This formula is the inequality estimating 


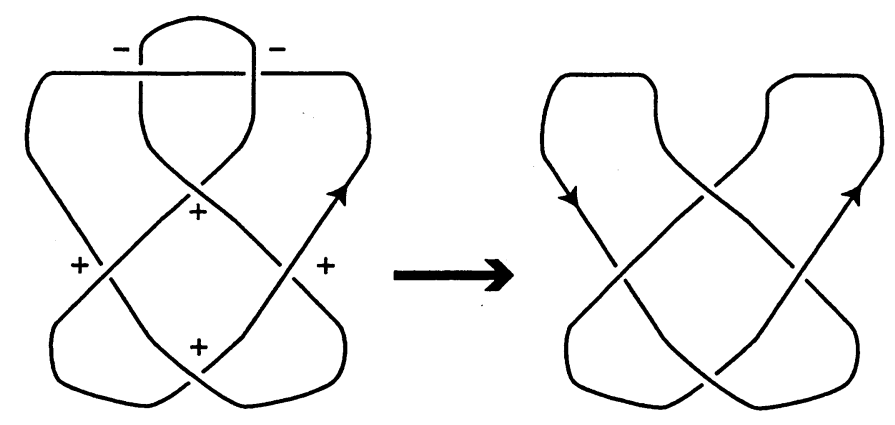

Figure 2. Construction of $D_{L}^{+}$from $D_{L}$

the genus of a closed oriented surface embedded in some closed 4-manifold and it was proved by Kronheimer, Mrowka, Morgan, Szabó, and Taubes [7], 8], 9], [12. The arguments due to Nakamura and Rudolph imply that the equality $\chi_{s}(L)=$ $s\left(D_{L}\right)-x\left(D_{L}\right)$ holds for any positive link diagram $D_{L}$, where $x\left(D_{L}\right)$ is the number of the crossings of $D_{L}$.

Proof of Theorem 3.1. Given an oriented link diagram $D$, let $\mathrm{O}(D)$ be the set of Seifert circles, and $\mathrm{X}(D)$ be the set of crossings. Let $\mathrm{X}_{>}(D)$ be the set of positive crossings, and $\mathrm{X}_{<}(D)$ be the set of negative crossings. Let $\mathrm{O}_{\geq}(D)$ be the set of non-negative circles, and $\mathrm{O}_{<}(D)$ be the set of strongly negative circles. We have $\mathrm{O}(D)=\mathrm{O}_{\geq}(D) \sqcup \mathrm{O}_{<}(D)$ and $\mathrm{X}(D)=\mathrm{X}_{>}(D) \sqcup \mathrm{X}_{<}(D)$. We denote by $\# A$ the number of elements of a set $A$.

Let $D_{L}^{+}$be a link diagram obtained from $D_{L}$ by putting

$$
\mathrm{O}\left(D_{L}^{+}\right):=\mathrm{O}_{\geq}\left(D_{L}\right) \subset \mathrm{O}\left(D_{L}\right), \mathrm{X}\left(D_{L}^{+}\right):=\mathrm{X}_{>}\left(D_{L}\right) \subset \mathrm{X}\left(D_{L}\right)
$$

and $L^{+}$be a link with $D_{L^{+}}=D_{L}^{+}$. For example, when $D_{L}$ is the link diagram on the left of Figure 2, let $D_{L}^{+}$be the link diagram illustrated on the right of Figure 2

First, we prove for the case $D_{L}^{+} \neq \emptyset$ by the argument due to Rudolph [18. The diagram $D_{L}^{+}$is not assumed to have crossings. When $D_{L}^{+} \neq \emptyset$, let $S$ and $Q$ be Seifert surfaces obtained by applying Seifert algorithm to $D_{L}$ and $D_{L}^{+}$respectively. We may suppose that $Q$ lies in the interior of $S$. Let $F$ be a smooth, oriented 2-manifold in $D^{4}$ without closed components such that $L=\partial F$ and $\chi(F)=\chi_{s}(L)$. The link diagram $D_{L}^{+}$is a positive link diagram. Hence we have $\chi_{s}\left(L^{+}\right)=\chi(Q)=$ $s\left(D_{L}^{+}\right)-x\left(D_{L}^{+}\right)$, which is a consequence from results by Nakamura [14] or Rudolph [16], [18]. We may suppose that $F \cup S$ is connected, since otherwise we can work separately for each component. Then, by same argument as the one in [17],

$$
\begin{aligned}
\chi(Q) & \geq \chi(F \cup S-Q) \\
& =\chi(F)+\chi(S)-\chi(Q),
\end{aligned}
$$

because we can regard $F \cup S-Q$ as a 2-manifold in the 4-ball with boundary $L^{+}$. Hence the inequality

$$
\chi_{s}(L) \leq 2 \chi(Q)-\chi(S)
$$


holds. So, therefore we have proved that the desired inequality holds in the case $D_{L}^{+} \neq \emptyset$ :

$$
\begin{aligned}
\chi_{s}(L) \leq & 2\left(\# \mathrm{O}\left(D_{L}^{+}\right)-\# \mathrm{X}\left(D_{L}^{+}\right)\right)-\left(\# \mathrm{O}\left(D_{L}\right)-\# \mathrm{X}\left(D_{L}\right)\right) \\
= & 2\left(s_{\geq}\left(D_{L}\right)-\# \mathrm{X}_{>}\left(D_{L}\right)\right) \\
& -\left\{\left(s_{\geq}\left(D_{L}\right)+s_{<}\left(D_{L}\right)\right)-\left(\# \mathrm{X}_{>}\left(D_{L}\right)+\# \mathrm{X}_{<}\left(D_{L}\right)\right)\right\} \\
= & \left(s_{\geq}\left(D_{L}\right)-s_{<}\left(D_{L}\right)\right)-w\left(D_{L}\right) .
\end{aligned}
$$

We add the proof for the case $D_{L}^{+}=\emptyset$. Let $L$ ! be the mirror image of the link $L$ and $D_{L}$ ! be the link diagram obtained by changing all crossings of $D_{L}$. When $D_{L}^{+}=\emptyset$, we have $D_{L}$ ! is a positive link diagram of $L$ !. Then

$$
\chi_{s}(L)=\chi_{s}(L !)=\# \mathrm{O}\left(D_{L} !\right)-\# \mathrm{X}\left(D_{L} !\right)
$$

as above. We have $\# \mathrm{X}\left(D_{L} !\right)=\# \mathrm{X}_{<}\left(D_{L}\right), \# \mathrm{O}\left(D_{L} !\right)=\# \mathrm{O}_{<}\left(D_{L}\right)=s_{<}\left(D_{L}\right)$, and $\# \mathrm{X}_{<}\left(D_{L}\right) \geq s_{<}\left(D_{L}\right)$ since each Seifert circle of $D_{L}$ is adjacent to at least two negative crossings. Since $D_{L}$ has no positive crossing, $w\left(D_{L}\right)=-\# \mathrm{X}_{<}\left(D_{L}\right)$. Hence we have

$$
\chi_{s}(L)=s_{<}\left(D_{L}\right)+w\left(D_{L}\right) \leq 0 \leq-s_{<}\left(D_{L}\right)-w\left(D_{L}\right) .
$$

Therefore we have proved that the desired inequality also holds in the case $D_{L}^{+}=\emptyset$ since $s_{\geq}\left(D_{L}\right)=0$.

Proof of Corollary 1.2. Theorem 3.1] implies that

$$
\begin{aligned}
u(L) \geq c_{s}(L) & \geq \frac{1}{2}\left(r-\chi_{s}(L)\right) \\
& \geq \frac{1}{2}\left\{r-\left(s_{\geq}\left(D_{L}\right)-s_{<}\left(D_{L}\right)\right)+w\left(D_{L}\right)\right\}
\end{aligned}
$$

by Theorem 1.1 .

To prove Theorem 1.3, we use the following lemma.

Lemma 3.2 (Boileau-Weber 3]). Let $L$ be an oriented link with $r$-components. We suppose that $L$ has a closed positive braid diagram $D_{L}$. Let $s\left(D_{L}\right)$ be the number of the Seifert circles and $x\left(D_{L}\right)$ the number of the crossings of $D_{L}$. Then the following inequality holds:

$$
u(L) \leq \frac{1}{2}\left(r-s\left(D_{L}\right)+x\left(D_{L}\right)\right) .
$$

Proof of Theorem 1.3. Since $D_{L}$ is a closed positive braid diagram, any Seifert circle of $D_{L}$ is non-negative, and $w\left(D_{L}\right)=x\left(D_{L}\right)$. Then

$$
u(L) \geq c_{s}(L) \geq \frac{1}{2}\left(r-s\left(D_{L}\right)+x\left(D_{L}\right)\right),
$$

by Corollary 1.2. Hence

$$
u(L)=c_{s}(L)=\frac{1}{2}\left(r-s\left(D_{L}\right)+x\left(D_{L}\right)\right),
$$

by Lemma 3.2 
Remark 3.3. Tanaka 21 determined the 4-genera and the unknotting numbers of some 10-crossing knots using a result of Rudolph [16], the equality $\chi_{s}(L)=$ $s\left(D_{L}\right)-w\left(D_{L}\right)$ for any quasipositive link diagram $D_{L}$. The author [5] determined the unknotting numbers of two 10-crossing knots using almost same as the argument in Auckly's lecture notes [1] based on the generalized adjunction formula proved by Kronheimer, Mrowka, Morgan, Szabó, and Taubes [9], 12.

Remark 3.4. Deformations of the link diagram by the Vogel algorithm and the Yamada algorithm do not change either the writhe or the number of Seifert circles. But they can change the value $\left(s_{\geq}-s_{<}\right)$.

\section{EXAmples}

In this section, we estimate $\chi_{s}$ or $u$ or $c_{s}$ for some links. We give two examples. One of them is the computation for torus links using Theorem 1.3. The other is the computation of lower bounds for the unknotting numbers and the 4-dimensional clasp numbers of the links obtained from certain links by fusion. It is an extension of a result by the author $[5$.

First, we give the following example.

Example 4.1. Let $T(p, q)$ be a torus link of type $(p, q)$. We suppose that $p$ and $q$ are positive integers. Let $d$ be the greatest common divisor of $p$ and $q$. Then

$$
u(T(p, q))=c_{s}(T(p, q))=\frac{1}{2}\{(p-1)(q-1)+d-1\} .
$$

Proof. The link $T(p, q)$ has $d$ components. By means of Theorem 1.3 we have

$$
u(T(p, q))=c_{s}(T(p, q))=\frac{1}{2}\{(p-1)(q-1)+d-1\}
$$

since $T(p, q)$ has a closed positive braid diagram with $p$ Seifert circles and $(p-1) q$ crossings.

In [10, Milnor conjectured that the unknotting number of the algebraic link is equal to the $\delta$-invariant of the singularity. The $\delta$-invariant is "the number of local double points" of the algebraic curve. This conjecture is true since it is a corollary of a result by Kronheimer and Mrowka [7, 8]. The above example gives an alternative proof of this conjecture for torus links. In fact, Milnor showed the equality $2 \delta=\mu+r-1$ in [10], where $\mu$ is the Milnor number of the singularity and $r$ is the number of components of the link. For the torus link of type $(p, q)$, we have $\mu=(p-1)(q-1)$, and $r=d$. Then $u(T(p, q))=c_{s}(T(p, q))=\delta$.

By Theorem 1.1 we extend a result by the author [5, Corollary 2.2]. In order to state it, we shall review the definition of fusion procedure, before estimating $\chi_{s}$ for a certain link using Theorem 3.1 .

Let $L$ be an oriented $r$-components link. Let $B_{1}, \cdots, B_{\nu}$ be mutually disjoint oriented bands in $S^{3}$ such that $B_{i} \cap L=\partial B_{i} \cap L=\alpha_{i} \cup \alpha_{i}^{\prime}$, where $\alpha_{1}, \alpha_{1}^{\prime}, \cdots, \alpha_{\nu}, \alpha_{\nu}^{\prime}$ are disjoint connected arcs. The closure of $L \cup \partial B_{1} \cup \cdots \cup B_{\nu}-\alpha_{1} \cup \alpha_{1}^{\prime} \cup \cdots \cup \alpha_{\nu} \cup \alpha_{\nu}^{\prime}$ is also the link. We will denote it by $L^{\prime}$. If $L^{\prime}$ has the orientation compatible with the orientation of $L-\bigcup_{i=1, \cdots, \nu} \alpha_{i} \cup \alpha_{i}^{\prime}$ and $\bigcup_{i=1, \cdots, \nu}\left(\partial B_{i}-\alpha_{i} \cup \alpha_{i}^{\prime}\right), L^{\prime}$ is called the link obtained from $L$ by the band surgery along the bands $B_{1}, \cdots, B_{\nu}$. Moreover if $L^{\prime}$ has $(r-\nu)$ components, this transformation is called a fusion.

Let $L=K_{1}^{1} \cup \cdots \cup K_{m_{1}+n_{1}}^{1} \cup K_{1}^{2} \cup \cdots \cup K_{m_{2}+n_{2}}^{2}$ be an $\left(\left(m_{1}+n_{1}, 0\right),\left(m_{2}+n_{2}, 0\right)\right)$ cable on the Hopf link with linking number 1 . We reverse orientations of $K_{m_{i}+j}^{i}$, 


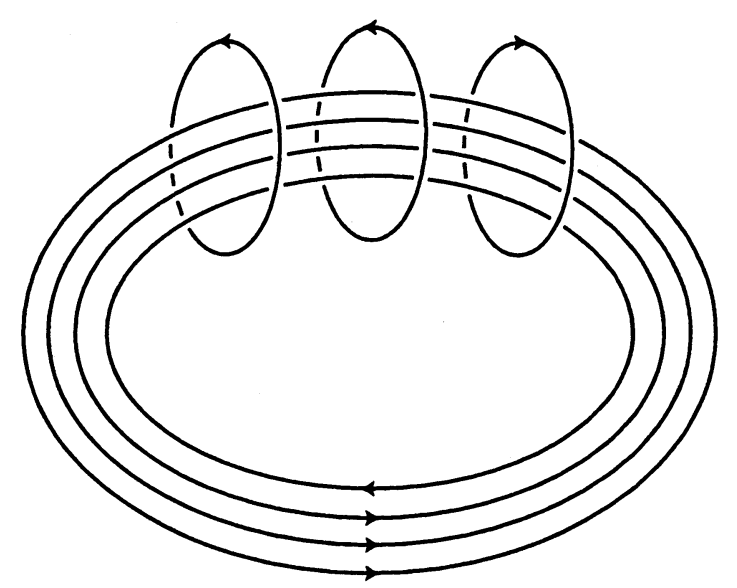

FIGURE 3. The link $L_{(3,1),(2,1)}$

$i=1,2, j=1, \cdots, n_{i}$, components of $L$. We denote these orientation reversed components by $L_{j}^{i}, i=1,2, j=1, \cdots, n_{i}$, and the link $K_{1}^{1} \cup \cdots \cup K_{m_{1}}^{1} \cup L_{1}^{1} \cup \cdots \cup$ $L_{n_{1}}^{1} \cup K_{1}^{2} \cup \cdots \cup K_{m_{2}}^{2} \cup L_{1}^{2} \cup \cdots \cup L_{n_{2}}^{2}$ by $L_{\left(m_{1}, n_{1}\right),\left(m_{2}, n_{2}\right)}$. For example, $L_{(3,1),(2,1)}$ is the link illustrated in Figure 3. We suppose $m_{1} \geq n_{1}, m_{2} \geq n_{2}$, and $m_{1}>0$.

Lemma 4.2. Let $L_{\left(m_{1}, n_{1}\right),\left(m_{2}, n_{2}\right)}$ be the above link. Then

$$
\chi_{s}\left(L_{\left(m_{1}, 0\right),\left(m_{2}, n_{2}\right)}\right)=m_{1}+m_{2}-n_{2}-2 m_{1}\left(m_{2}-n_{2}\right) .
$$

Proof. We consider the trivial solid torus $V=S^{1} \times D^{2}$ in $S^{3}$. We suppose that $K_{1}^{1}, \cdots, K_{m_{1}}^{1}$ lie parallel to $S^{1} \times\{*\}$ in the interior of $V$, and that $K_{1}^{2}, \cdots, K_{m_{2}}^{2}$, $L_{1}^{2}, \cdots, L_{n_{2}}^{2}$ lie parallel to $\{*\} \times \partial D^{2}$ on $\partial V$. We consider the trivial diagram for $V$. This condition gives the link diagram $D_{L_{\left(m_{1}, 0\right),\left(m_{2}, n_{2}\right)}}$ as in Figure 3, It has $\left(m_{1}+m_{2}\right)$ non-negative circles, $n_{2}$ strongly negative circles, $2 m_{1} m_{2}$ positive crossings, and $2 m_{1} n_{2}$ negative crossings. Applying Theorem 3.1, we have

$$
\chi_{s}\left(L_{\left(m_{1}, 0\right),\left(m_{2}, n_{2}\right)}\right) \leq m_{1}+m_{2}-n_{2}-2 m_{1}\left(m_{2}-n_{2}\right) .
$$

In order to prove the equality, we construct a 2-manifold $F$ embedded in $S^{3} \times$ $[0,1]=\partial D^{4} \times[0,1] \subset D^{4}$ such that $\partial F=L_{\left(m_{1}, 0\right),\left(m_{2}, n_{2}\right)}$ and $\chi(F)=m_{1}+$ $m_{2}-n_{2}-2 m_{1}\left(m_{2}-n_{2}\right)$. We can suppose that each $K_{m_{2}-n_{2}+j}^{2}$ lies in the tubular neighborhood of $L_{j}^{2}$. Let $B_{j}$ be a trivial band connecting $K_{m_{2}-n_{2}+j}^{2}$ and $L_{j}^{2}$ in the above tubular neighborhood. The link obtained from $L_{\left(m_{1}, 0\right),\left(m_{2}, n_{2}\right)}$ by the band surgery along the bands $B_{1}, \cdots, B_{n_{2}}$ is the link $L_{\left(m_{1}, 0\right),\left(m_{2}-n_{2}, 0\right)} \cup O_{1} \cup \cdots \cup O_{n_{2}}$ where each $O_{j}$ is a split trivial component. We identify $F \cap\left(S^{3} \times\{t\}\right)$ with this band surgery in $S^{3}$ at time $t(t \in[0,2 / 3])$. Here we put

$$
\begin{aligned}
F \cap\left(S^{3} \times\{0\}\right) & =L_{\left(m_{1}, 0\right),\left(m_{2}, n_{2}\right)} \times\{0\} \\
F \cap\left(S^{3} \times\left\{\frac{1}{3}\right\}\right) & =\left(L_{\left(m_{1}, 0\right),\left(m_{2}, n_{2}\right)} \cup B_{1} \cup \cdots \cup B_{n_{2}}\right) \times\left\{\frac{1}{3}\right\} \\
F \cap\left(S^{3} \times\left\{\frac{2}{3}\right\}\right) & =\left(L_{\left(m_{1}, 0\right),\left(m_{2}-n_{2}, 0\right)} \cup O_{1} \cup \cdots \cup O_{n_{2}}\right) \times\left\{\frac{2}{3}\right\} .
\end{aligned}
$$

Let $S$ be a Seifert surface with boundary $L_{\left(m_{1}, 0\right),\left(m_{2}-n_{2}, 0\right)}$, obtained by applying the Seifert algorithm to the link diagram similar to the one in the proof of the above 
inequality. Let $D_{j}$ 's be the disjoint disks in $S^{3}-L_{\left(m_{1}, 0\right),\left(m_{2}-n_{2}, 0\right)}$ with $\partial D_{j}=O_{j}$. We put

$$
F \cap\left(S^{3} \times\{1\}\right)=\left(S \cup D_{1} \cup \cdots \cup D_{n_{2}}\right) \times\{1\} \subset S^{3} \times[0,1] \subset D^{4} .
$$

Thus we obtain the desired surface $F$. Then

$$
\begin{aligned}
\chi_{s}\left(L_{\left(m_{1}, 0\right),\left(m_{2}, n_{2}\right)}\right) & \geq \chi(F) \\
& =\chi(S)+n_{2} \chi(\text { annulus }) \\
& =m_{1}+m_{2}-n_{2}-2 m_{1}\left(m_{2}-n_{2}\right) .
\end{aligned}
$$

Hence the equality holds.

Theorem 4.3. Let $L_{\left(m_{1}, n_{1}\right),\left(m_{2}, n_{2}\right)}$ be the above link. If an oriented $r$-components link $L$ in $S^{3}$ is obtained from $L_{\left(m_{1}, 0\right),\left(m_{2}, n_{2}\right)}$ by the fusion, then

$$
u(L) \geq c_{s}(L) \geq m_{1}\left(m_{2}-n_{2}\right)-\left(m_{1}+m_{2}\right)+r .
$$

Proof. By constructing a 2-manifold with boundary $L_{\left(m_{1}, 0\right),\left(m_{2}, n_{2}\right)}$ from the fusion, we have

$$
\chi_{s}\left(L_{\left(m_{1}, 0\right),\left(m_{2}, n_{2}\right)}\right) \geq \chi_{s}(L)-\left(m_{1}+m_{2}+n_{2}-r\right) .
$$

By Lemma 4.2 we have

$$
\chi_{s}(L) \leq 2\left(m_{1}+m_{2}\right)-2 m_{1}\left(m_{2}-n_{2}\right)-r .
$$

By Theorem 1.1,

$$
\begin{aligned}
u(L) \geq c_{s}(L) & \geq \frac{1}{2}\left(r-\chi_{s}(L)\right) \\
& \geq m_{1}\left(m_{2}-n_{2}\right)-\left(m_{1}+m_{2}\right)+r .
\end{aligned}
$$

If $r=1$, the above result can be proved by using the argument in [6], which implies the following inequality.

Theorem 4.4 ([6]). Let $L_{\left(m_{1}, n_{1}\right),\left(m_{2}, n_{2}\right)}$ be the above link. If an oriented knot $K$ in $S^{3}$ is obtained from $L_{\left(m_{1}, n_{1}\right),\left(m_{2}, n_{2}\right)}$ by the band surgery along the $\nu$ bands, then

$$
u(K) \geq c_{s}(K) \geq\left(m_{1}-n_{1}\right)\left(m_{2}-n_{2}\right)-\frac{1}{2}\left(\nu+m_{1}-n_{1}+m_{2}-n_{2}-1\right) .
$$

Remark 4.5. By means of a result by Miyazaki and Yasuhara 11, Proposition 3.6], there is a 2-components link which cannot be obtained from $L_{\left(m_{1}, n_{1}\right),\left(m_{2}, n_{2}\right)}$ by the fusion.

\section{ACKNOWLEDGMENT}

The author would like to thank Professor Toshitake Kohno for his encouragement. She also would like to thank Professor Hitoshi Murakami and Professor Akira Yasuhara for their useful comments and Mr. Hiroshi Matsuda for his helpful suggestions and comments about the Bennequin unknotting inequality. 


\section{REFERENCES}

1. D. Auckly, Surgery, knots, and the Seiberg-Witten equations, Lectures for the 1995 TGRCIW, preprint.

2. D. Bennequin, Entrelacement et équations de Pfaff, Astérisque 107-108 (1983), 87-161. MR 86e:58070

3. M. Boileau and C. Weber, Le problème de J. Milnor sur le nombre gordien des nouds algébriques, Enseign. Math. 30 (1984), 173-222. MR 86c:57004

4. R. Gompf, Smooth concordance of topologically slice knots, Topology 25 (1986), 353-373. MR 87i:57004

5. T. Kawamura, The unknotting numbers of $10_{139}$ and $10_{152}$ are 4, Osaka J. Math. 35 (1998), 539-546. MR 99k:57018

6. L Lower bounds for the unknotting numbers of the knots obtained from certain links, Knots in Hellas '98: Proc. of the International Conference on Knot Theory and Its Ramifications (C. Gordon, et al., eds.), Ser. Knots Everything 24, World Sci. Publ., Singapore, 2000, 203-207.

7. P. Kronheimer and T. Mrowka, Gauge theory for embedded surfaces, I, Topology 32 (1993), 773-826. MR 94k:57048

8. _ Gauge theory for embedded surfaces, II, Topology 34 (1995), 37-97. MR 96b:57038

9. 797-808. MR 96a:57073

10. J. Milnor, Singular points of complex hypersurfaces, Ann. of Math. Studies 61 (1968), Princeton Univ. Press. MR 39:969

11. K. Miyazaki and A. Yasuhara, Generalized \#-unknotting operations, J. Math. Soc. Japan 49 (1997), 107-123. MR 97i:57007

12. J. Morgan, Z. Szabó and C. Taubes, A product formula for the Seiberg-Witten invariants and the generalized Thom conjecture, J. Differential Geom. 44 (1996), 706-788. MR 97m:57052

13. H. Murakami and A. Yasuhara, Four-genus and four-dimensional clasp number of a knot, Proc. Amer. Math. Soc. 128 (2000), 3693-3699. MR 2001b:57020

14. T. Nakamura, Four-genus and unknotting number of positive knots and links, Osaka J. Math 37 (2000), 441-452. MR 2001e:57005

15. L. Rudolph, Algebraic functions and closed braids, Topology 22 (1983), 191-201. MR 84e:57012

16. 51-59. MR 94d:57028

17. - Quasipositive plumbing (Constructions of quasipositive knots and links, V), Proc. Amer. Math. Soc. 126 (1998), 257-267. MR 98h:57024

18. - Positive links are strongly quasipositive, Geometry and Topology Monographs 2 (1999): Proc. of the Kirbyfest, 555-562. MR 2000j:57015

19. T. Shibuya, Some relations among various numerical invariants for links, Osaka J. Math. 11 (1974), 313-322. MR 50:5779

20. A. Stoimenow, Positive knots, closed braids and the Jones polynomial, preprint (1999).

21. T. Tanaka, Unknotting numbers of quasipositive knots, Topology Appl. 88 (1998), 239-246. MR 99g:57014

22. P. Vogel, Representation of links by braids: A new algorithm, Comment. Math. Helv. 65 (1990), 104-113. MR 90k:57013

23. S. Yamada, The minimal number of Seifert circles equals the braid index of a link, Invent. Math. 89 (1987), 347-356. MR 88f:57015

Graduate School of Mathematical Sciences, University of Tokyo, 3-8-1 Komaba Meguro-Ku, TOKYO 153-8914, JAPAN

E-mail address: kawamura@ms.u-tokyo.ac.jp 